Bull. Korean Math. Soc. 52 (2015), No. 2, pp. 679-684

http://dx.doi.org/10.4134/BKMS.2015.52.2.679

\title{
SOME REMARKS ON TOTAL CURVATURE OF A MINIMAL GRAPH
}

\author{
AndRZej GancZar
}

\begin{abstract}
In this paper we discuss bounds for the total curvature of nonparametric minimal surfaces by using the properties of planar harmonic mappings.
\end{abstract}

\section{Introduction}

A complex-valued function $f(z)=u(z)+i v(z)$, defined on some domain $D \subset \mathbb{C}$ is a planar harmonic mapping if the components $u$ and $v$ are real-valued harmonic functions which need not be conjugate. Throughout this article we will discuss harmonic mappings of the unit disk $\mathbb{D}=\{z \in \mathbb{C}:|z|<1\}$. A harmonic mapping $f$ defined on $\mathbb{D}$ can be uniquely written as $f=h+\bar{g}$, $g(0)=0$, where $h$ and $g$ belong to the linear space $\mathcal{H}(\mathbb{D})$ of all holomorphic functions on $\mathbb{D}$. The mapping $f$ is locally univalent if and only if its Jacobian $\left|h^{\prime}\right|^{2}-\left|g^{\prime}\right|^{2}$ does not vanish. If we require that $f$ is orientation-preserving, then the second complex dilatation $\omega(z)=g^{\prime}(z) / h^{\prime}(z)$ belongs to $\mathcal{H}(\mathbb{D})$ and $|\omega(z)|<1$ on $\mathbb{D}$. References for this material include [2] and [5].

Harmonic univalent mappings were first studied in connection with minimal surfaces by E. Heinz (see [7]). This relationship between a univalent harmonic mapping and a minimal graph $\mathcal{M}$ comes from conformal representation of $\mathcal{M}$ via the Weierstrass-Enneper formulas (see e.g. [4]). Let $\mathcal{M}=\{(u, v, F(u, v))$ : $(u, v) \in \Omega\}$ be a nonparametric surface lying over a simply connected proper subdomain $\Omega$ of the complex plane $\mathbb{C}$. If $\mathcal{M}$ is parametrized by orientationpreserving isothermal parameters $z=x+i y \in \mathbb{D}$, the projection onto its base plane gives a univalent harmonic mapping $f(z)=u+i v$ of $\mathbb{D}$ onto $\Omega$ whose dilatation $\omega$ is the square of an holomorphic function with $|\omega(z)|<1$ on $\mathbb{D}$. Conversely, if $f=h+\bar{g}$ is an orientation-preserving univalent harmonic mapping of $\mathbb{D}$ onto $\Omega$ with dilatation $\omega=p^{2}$ for some function $p \in \mathcal{H}(\mathbb{D})$, then

Received May 26, 2014; Revised July 8, 2014.

2010 Mathematics Subject Classification. Primary 30C45, $30 \mathrm{C} 55$.

Key words and phrases. minimal surface, univalent harmonic mapping, dilatation, Bloch function. 
the formulas

$$
u(z)=\operatorname{Re}\{f(z)\}, \quad v(z)=\operatorname{Im}\{f(z)\}, \quad F(z)=2 \operatorname{Im}\left\{\int_{z_{0}}^{z} h^{\prime}(\zeta) p(\zeta) d \zeta\right\}
$$

define by isothermal parameters a nonparametric minimal surface $\mathcal{M}$ whose projection is $f$, so the first fundamental form of $\mathcal{M}$ is $d s^{2}=\lambda^{2}|d z|^{2}$, where $\lambda=\left|h^{\prime}\right|+\left|g^{\prime}\right|$ is the conformal metric. Recall, that the general formula for Gauss curvature is

$$
K=-\frac{1}{\lambda^{2}} \Delta(\log \lambda),
$$

where $\Delta$ denotes the Laplacian. Therefore, in terms of the harmonic mapping $f=h+\bar{g}$, the Gauss curvature is found to be

$$
K(\xi)=-\frac{\left|\omega^{\prime}(z)\right|^{2}}{\left|h^{\prime}(z) g^{\prime}(z)\right|(1+|\omega(z)|)^{4}}, \quad z \in \mathbb{D}
$$

at a point $\xi \in \mathcal{M}$ that lies above $f(z)$. The equality $\omega=p^{2}$ implies that

$$
K=-\frac{4\left|p^{\prime}\right|^{2}}{\left(\left|h^{\prime}\right|+\left|g^{\prime}\right|\right)^{2}\left(1+|p|^{2}\right)^{2}} .
$$

\section{Main results}

Suppose that $\mathcal{M}$ is a minimal graph given by orientation-preserving isothermal parameters over a simply connected domain $\Omega, \Omega \subset \mathbb{C}$, and $f: \mathbb{D} \rightarrow \Omega$ is an orientation-preserving univalent harmonic mapping with dilatation $\omega=p^{2}$ corresponding to $\mathcal{M}$. If we now let $\mathcal{G}$ to be a domain whose closure is in $\mathbb{D}$, the surface over $f(\mathcal{G}) \subset \Omega$ has total curvature $T_{f, p}(\mathcal{G})$ given by

$$
T_{f, p}(\mathcal{G})=\iint_{\mathcal{G}} K \lambda^{2} d A(z), \quad z=x+i y,
$$

where $d A(z)=d x d y$ is the Euclidean area element. From (1) and (2) we obtain

$$
T_{f, p}(\mathcal{G})=-4 \iint_{\mathcal{G}} \frac{\left|p^{\prime}(z)\right|^{2}}{\left(1+|p(z)|^{2}\right)^{2}} d A(z),
$$

which is due to the fact that $f$ has dilatation $\omega=p^{2}$. It should be noted that $p^{\sharp}(z)=\frac{\left|p^{\prime}(z)\right|}{1+|p(z)|^{2}}$ is the spherical derivative of $p$, and consequently $\frac{1}{4}\left|T_{f, p}(\mathcal{G})\right|$ is the area of the spherical image of $p(\mathcal{G})$ counted with multiplicity. By the above $\left|T_{f, p}(\mathcal{G})\right| \geq 4 \mathcal{A}_{s}(p(\mathcal{G}))$, where $\mathcal{A}_{s}(p(\mathcal{G}))$ is the spherical area of the set $p(\mathcal{G})$ identified with its projection to the Riemann sphere.

Since $p$ is a holomorphic function mapping $\mathbb{D}$ into itself, the Schwarz-Pick lemma gives

$$
\left|T_{f, p}(\mathcal{G})\right| \leq 4 \iint_{\mathcal{G}}\left(\frac{1-|p(z)|^{2}}{1+|p(z)|^{2}}\right)^{2} \frac{d A(z)}{\left(1-|z|^{2}\right)^{2}} \leq 4 \iint_{\mathcal{G}} \frac{d A(z)}{\left(1-|z|^{2}\right)^{2}}
$$

as is easy to check. An estimate due to Goluzin yields a refinement of the above inequality. 
Lemma 2.1. Suppose that $p \in \mathcal{H}(\mathbb{D})$ has the expansion $p(z)=a_{0}+\sum_{n=k}^{\infty} a_{n} z^{n}$, where $k \geq 1$, and $f$ is an orientation-preserving univalent harmonic mapping with dilatation $\omega=p^{2}$. Then

$$
\left|T_{f, p}(\mathcal{G})\right| \leq 4 k^{2} \iint_{\mathcal{G}} \frac{|z|^{2 k-2}}{\left(1-|z|^{2 k}\right)^{2}} d A(z) .
$$

Proof. We have used only the fact (see [6, page 333]) that

$$
\left(1-|z|^{2 k}\right)\left|p^{\prime}(z)\right| \leq k|z|^{k-1}\left(1-|p(z)|^{2}\right), \quad z \in \mathbb{D}
$$

for a function $p(z)=a_{0}+\sum_{n=k}^{\infty} a_{n} z^{n}$, where $k \geq 1$, holomorphic and $|p(z)|<1$ on $\mathbb{D}$.

Given any $r \in(0,1)$ suppose that $D_{r}=\{z \in \mathbb{C}:|z|<r\}$. Taking $\mathcal{G}=D_{r}$ we can now rephrase Lemma 2.1 as follows.

Corollary 2.2. For any $r \in(0,1)$ we have

$$
\left|T_{f, p}\left(D_{r}\right)\right| \leq 4 \pi \frac{r^{2}}{1-r^{2}}
$$

Theorem 2.3. Let $f$ be a harmonic univalent mapping of $\mathbb{D}$ with dilatation $\omega=p^{2}$, where $p \in \mathcal{H}(\mathbb{D}), p(\mathbb{D}) \subset \mathbb{D}$ and $p(0)=0$. If $r^{2} \in\left[\frac{m-1}{m}, \frac{m}{m+1}\right]$, where $m \in \mathbb{N}$, then

$$
\left|T_{f, p}\left(D_{r}\right)\right| \leq 4 m \pi r^{2 m}
$$

Proof. Assume that $f$ has dilatation $\omega=p^{2}$ and observe that

$$
\left|T_{f, p}\left(D_{r}\right)\right| \leq 4 \iint_{D_{r}}\left|p^{\prime}(z)\right|^{2} d A(z)=4 \int_{0}^{2 \pi} \int_{0}^{r}\left|p^{\prime}\left(\rho e^{i \theta}\right)\right| \rho d \rho d \theta
$$

which is clear from (3). Then the assertion follows from [8, Corollary on page $262]$, since $p$ is subordinate to the identity function $I(z)=z$.

In particular we obtain:

Corollary 2.4. Under the assumptions of Theorem 2.3, we have

$$
\left|T_{f, p}\left(D_{r}\right)\right| \leq \frac{4 \pi}{e}(m+1), \quad r^{2} \in\left[\frac{m-1}{m}, \frac{m}{m+1}\right]
$$

for all $m \in \mathbb{N}$.

Proof. Theorem 2.3 yields $\left|T_{f, p}\left(D_{r}\right)\right| \leq 4 \pi(m+1)\left(\frac{m}{m+1}\right)^{m+1}$ for all $\frac{m-1}{m} \leq$ $r^{2} \leq \frac{m}{m+1}$. To prove the result we note that $\left(\left(\frac{m}{m+1}\right)^{m+1}\right)_{m \in \mathbb{N}}$ is an increasing sequence and $\lim _{m \rightarrow \infty}\left(\frac{m}{m+1}\right)^{m+1}=e^{-1}$.

Theorem 2.5. Suppose that $f$ has the dilatation $\omega=p^{2}$, where $p \in \mathcal{H}(\mathbb{D})$, $p(0)=0$ and $|p(z)|<1$ on $\mathbb{D}$. For any fixed $r \in(0,1)$ we have

$$
A_{f, p}(r)=\int_{0}^{r} \frac{\left|T_{f, p}\left(D_{t}\right)\right|}{t} d t \leq 2 \pi \log \left(1+r^{2}\right) .
$$


Equality holds if $p$ is a function of the form $p(z)=e^{i \theta} z, \theta \in \mathbb{R}$. In particular, for any $r \in(0,1)$ we have $A_{f, p}(r)<2 \pi \log 2$.

Proof. We have

$$
\Delta \log \left(1+|p(z)|^{2}\right)=\frac{4\left|p^{\prime}(z)\right|^{2}}{\left(1+|p(z)|^{2}\right)^{2}}
$$

where $\Delta$ denotes the Laplacian in the $z$-coordinate. On the account of this, an application of Green's theorem gives

$$
\left|T_{f, p}\left(D_{r}\right)\right|=\int_{|z|=r} \frac{\partial}{\partial n} \log \left(1+|p(z)|^{2}\right) d s=r \frac{\partial}{\partial r} \int_{0}^{2 \pi} \log \left(1+\left|p\left(r e^{i \theta}\right)\right|^{2}\right) d \theta,
$$

and for $r \in(0,1)$ we get

$$
\int_{0}^{r} \frac{\left|T_{f, p}\left(D_{t}\right)\right|}{t} d t=\int_{0}^{2 \pi} \log \left(1+\left|p\left(r e^{i \theta}\right)\right|^{2}\right) d \theta-2 \pi \log \left(1+|p(0)|^{2}\right) .
$$

The Schwarz lemma enables us to write $|p(z)| \leq|z|$ on $\mathbb{D}$, and finally

$$
\int_{0}^{r} \frac{\left|T_{f, p}\left(D_{t}\right)\right|}{t} d t \leq \int_{0}^{2 \pi} \log \left(1+r^{2}\right) d \theta=2 \pi \log \left(1+r^{2}\right)<2 \pi \log 2
$$

for a fixed $r \in(0,1)$.

Now, we wish to investigate the case when $p$ is a Bloch function. This means that the Bloch constant $\beta_{p}=\sup \left\{\left(1-|z|^{2}\right)\left|p^{\prime}(z)\right|: z \in \mathbb{D}\right\}$ is finite. Assume that $f$ is an orientation-preserving univalent harmonic mapping of $\mathbb{D}$ and has a dilation $\omega=p^{2}$, where $p$ is a Bloch function. Since $p(\mathbb{D}) \subset \mathbb{D}$, the constant $\beta_{p}$ is no greater than one. This clearly forces

$$
\left|T_{f, p}\left(D_{r}\right)\right| \leq 4 \pi \beta_{p}^{2} \frac{r^{2}}{1-r^{2}}, \quad r \in(0,1) .
$$

Theorem 2.6. If $f$ is an orientation-preserving univalent harmonic mapping with dilatation $\omega=p^{2}$, where $p$ is a non-vanishing inner function or an outer function, then

$$
\left|T_{f, p}\left(D_{r}\right)\right| \leq \frac{16 \pi}{e^{2}} \frac{r^{2}}{1-r^{2}}, \quad r \in(0,1) .
$$

Proof. In the case we have $\beta_{p}=\frac{2}{e}$ (see [3, Theorem 5]).

Given $a \in \mathbb{D}$, let $\varphi_{a}(z)=(a-z) /(1-\bar{a} z)$ be a Möbius transformation of $\mathbb{D}$, and let $\Delta(a, r)=\left\{z \in \mathbb{D}:\left|\varphi_{a}(z)\right|<r\right\}$ be the pseudohyperbolic disk with center $a$ and radius $r \in(0,1)$. The composition $F_{a}=f \circ \varphi_{a}$ is again univalent harmonic and orientation-preserving on $\mathbb{D}$, but the important thing is that $F_{a}$ has dilatation $\omega \circ \varphi_{a}=\left(p \circ \varphi_{a}\right)^{2}$. Therefore, we can consider a nonparametric minimal surface $\mathcal{M}_{a}$ corresponding to $F_{a}$ for a fixed $a \in \mathbb{D}$. Then for a fixed $r \in(0,1)$ we have

$$
A_{F_{a}, p \circ \varphi_{a}}(r)=4 \int_{0}^{r} \frac{d t}{t} \iint_{D_{t}}\left[\left(p \circ \varphi_{a}\right)^{\sharp}\right]^{2} d A(z)
$$




$$
\begin{aligned}
& =4 \int_{0}^{r} \frac{d t}{t} \iint_{\Delta(a, t)}\left[p^{\sharp}(z)\right]^{2} d A(z) \\
& \leq 4 \beta_{p}^{2} \int_{0}^{r} \frac{d t}{t} \iint_{\Delta(a, t)} \frac{d A(z)}{\left(1-|z|^{2}\right)^{2}} \\
& =2 \pi \beta_{p}^{2} \log \frac{1}{1-r^{2}},
\end{aligned}
$$

provided that $p$ is a Bloch function. In particular, given $r \in(0,1)$ we have $\sup _{a \in \mathbb{D}} A_{F_{a}, p \circ \varphi_{a}}(r)<\infty$.

\section{Remarks}

It follows from $\left[9\right.$, Theorem 1] that $p^{\sharp}(z) \leq \sqrt{\frac{\beta}{\pi-\beta}}\left(1-|z|^{2}\right)^{-1}$ at each $z \in \mathbb{D}$, provided $\mathcal{A}_{s}(p(\mathbb{D})) \leq \beta<\pi$. Note that in our case we have $\mathcal{A}_{s}(p(\mathbb{D})) \leq \beta \leq \frac{\pi}{2}$, since $p(\mathbb{D}) \subset \mathbb{D}$, and this gives

$$
\left|T_{f, p}\left(D_{r}\right)\right| \leq \frac{4 \pi \beta}{\pi-\beta} \frac{r^{2}}{1-r^{2}}, \quad r \in(0,1) .
$$

Assume that $p$ is not necessarily Bloch and $\sup _{a \in \mathbb{D}} A_{F_{a}, p \circ \varphi_{a}}(r)<\infty$ for some $r \in(0,1)$. Then exists $r_{*} \in(0, r)$ such that

$$
\sup _{a \in \mathbb{D}} A_{F_{a}, p \circ \varphi_{a}}(r)<\log \frac{r}{r_{*}}
$$

and according to the above

$$
\begin{aligned}
A_{F_{a}, p \circ \varphi_{a}}(r) & \geq 4 \int_{r_{*}}^{r} \frac{d t}{t} \iint_{\Delta(a, t)}\left[p^{\sharp}(z)\right]^{2} d A(z) \\
& \geq 4\left(\log \frac{r}{r_{*}}\right) \iint_{\Delta\left(a, r_{*}\right)}\left[p^{\sharp}(z)\right]^{2} d A(z),
\end{aligned}
$$

which yields

$$
\sup _{a \in \mathbb{D}}\left|T_{f, p}\left(\Delta\left(a, r_{*}\right)\right)\right| \leq\left(\log \frac{r}{r_{*}}\right)^{-1} \sup _{a \in \mathbb{D}} A_{F_{a}, p \circ \varphi_{a}}(r)<1 .
$$

According to $\left[3\right.$, Theorem 6], if $p: \mathbb{D} \rightarrow \mathbb{D}$ is holomorphic on $\mathbb{D}$ with $\beta_{p}=1$ then either $p$ is conformal automorphism of $\mathbb{D}$ or all zeros of $p$ define a convergent infinite Blaschke product $B$ with $\beta_{B}=1$.

If $p$ is finite Blaschke product of degree greater than 1 , then $\beta_{p}<1$ (see [3, Corollary on page 96]) and (5) is better than the bound given in Corollary 2.2. If, in addition, $f(\mathbb{D})$ is a simple bounded polygonal domain, then $\mathcal{M}$ is a Jenkins-Serrin surface (see e.g. [1]). 


\section{References}

[1] D. Bshouty and A. Weitsman, On the Gauss map of minimal graphs, Complex Var. Theory Appl. 48 (2003), no. 4, 339-346.

[2] J. G. Clunie and T. Sheil-Small, Harmonic univalent functions, Ann. Acad. Sci. Fenn. Ser. A I 9 (1984), 3-25.

[3] F. Colonna, The Bloch constant of bounded analytic functions, J. London Math. Soc. 36 (1987), no. 1, 95-101.

[4] U. Dierkes, S. Hildebrandt, A. Küster, and O. Wohlrab, Minimal Surfaces I. Boundary Value Problems, Grundlehren der Math. Wiss. 295, Springer-Verlag, 1992.

5] P. Duren, Harmonic Mappings in the Plane, Cambridge Tracts in Mathematics vol. 156, Cambridge University Press, 2004.

[6] G. M. Goluzin, Geometric Theory of Functions of a Complex Variable, GITTL, Moscow, 1952; English transl., Transl. Math. Monographs, vol. 26, Amer. Soc. Providence, R.I., 1969.

[7] E. Heinz, Über die Losungen der Minimalflachengleichung, Nachr. Akad. Wiss. G̈̈tingen. Math.-Phys. Kl. Math.-Phys.-Chem. Abt. (1952), 51-56.

[8] E. Reich, An inequality for subordinate analytic functions, Pacific J. Math. 4 (1954), no. 2, 259-274.

[9] S. Yamashita, Spherical derivative of meromorphic function with image of finite spherical area, J. Inequal. Appl. 5 (2000), no. 2, 191-199.

INSTITUTE OF MATHEMATICS

Maria Curie-SkŁodowska University

Pl. Marit Curie-SkŁodowskiej 1

20-031 Lublin, POland

E-mail address: aganczar@hektor.umcs.lublin.pl 